Commun. Fac. Sci. Univ. Ank. Ser. A1 Math. Stat.

Volume 68, Number 2, Pages 21542160(2019)

DOI: $10.31801 /$ cfsuasmas. 510560

ISSN 1303-5991 E-ISSN 2618-6470

http://communications.science.ankara.edu.tr/index.php?series=A1

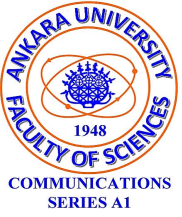

\title{
ON SOME APPROXIMATION PROPERTIES OF THE GAUSS-WEIERSTRASS OPERATORS
}

\author{
BAŞAR YILMAZ
}

\begin{abstract}
In this paper, we present some approximation properties of the Gauss-Weierstrass operators in exponential weighted spaces including norm convergence of them and Voronovskaya and quantitative Voronovskaya-type theorems.
\end{abstract}

\section{Preliminaries}

The Gauss-Weierstrass singular integral operator

$$
\left(W_{n} f\right)(x):=\sqrt{\frac{n}{\pi}} \int_{-\infty}^{\infty} f(x+t) e^{-n t^{2}} d t,
$$

where $x \in \mathbb{R}, n \in \mathbb{N}$ and $n \rightarrow \infty$, was examined in [1, 3], 4], 8] for functions belonging to the space $L_{p}$ and the classical Hölder spaces.

In this paper we examine the Gauss-Weierstrass operators $W_{n}$ for functions $f$ belonging to the exponential weighted spaces $L_{q}^{p}(\mathbb{R})$ and $L_{q}^{p, r}(\mathbb{R})$ which definitions are given bellow. We give some elementary properties, the orders of approximation and the Voronovskaya type theorem and quantitative Voronovskaya type theorem for these operators. Also simultaneous approximation property is obtained.

Let $q>0$ be a fixed number and let

$$
\nu_{q}(x):=e^{-q x^{2}}, \quad x \in \mathbb{R} \text {. }
$$

For a fixed $1 \leq p \leq \infty$ and $q>0$ we denote by $L_{q}^{p}$ the set of all real-valued functions $f$ defined on $\mathbb{R}$ for which the $p$ - th power of $\nu_{q} f$ is Lebesgue-integrable on $\mathbb{R}$ if $1 \leq p<\infty$, and $\nu_{q} f$ is uniformly continuous and bounded on $\mathbb{R}$ if $p=\infty$. Let the norm in $L_{q}^{p}$ be given below by the formula

2010 Mathematics Subject Classification. Primary 41A36, Secondary 41A25.

Key words and phrases. Gauss-Weierstrass operators, Voronovskaya type theorem, exponential weighted space.

(C)2019 Ankara University Communications Faculty of Sciences University of Ankara-Series A1 Mathematics and Statistics 


$$
\|f\|_{p, q}=\|f(.)\|_{p, q}:= \begin{cases}\left(\int_{-\infty}^{\infty}\left|\nu_{q}(x) f(x)\right|^{p} d x\right)^{1 / p}, & \text { if } 1 \leq p<\infty, \\ \sup _{x \in \mathbb{R}} \nu_{q}(x)|f(x)| & \text { if } p=\infty .\end{cases}
$$

Also, let $r \in \mathbb{N}_{0}$ and $L_{q}^{p, r} \equiv L_{q}^{p, r}(\mathbb{R})$ be the class of all $r$-times differentiable functions $f \in L_{q}^{p}$ having the derivatives $f^{(k)} \in L_{q}^{p}, 1 \leq k \leq r$. The norm in $L_{q}^{p, r}$ is given by (3). The spaces $L_{q}^{p}$ and $L_{q}^{p, r}$ are called exponential weighted spaces(see [2]).

For $f \in L_{q}^{p}$ we define the modulus of smoothness of the order two (see [5])

$$
\omega_{2}\left(f, L_{q}^{p} ; t\right):=\sup _{|h| \leq t}\left\|\Delta_{h}^{2} f(\cdot)\right\|_{p, q} \text { for } t \geq 0
$$

where

$$
\Delta_{h}^{2} f(x):=f(x+h)-f(x-h)-2 f(x), \quad x, h \in \mathbb{R}
$$

From $(3)-(5)$ for $f \in L_{q}^{p}$ follows

$$
\begin{gathered}
\|f(\cdot+h)\|_{p, q} \leq e^{q h^{2}}\|f(\cdot)\|_{p, q}, \quad h \in \mathbb{R}, \\
0=\omega_{2}\left(f ; L_{p}^{q} ; 0\right) \leq \omega_{2}\left(f, L_{p}^{q} ; t_{1}\right) \leq \omega_{2}\left(f, L_{p}^{q} ; t_{2}\right) \text { if } 0 \leq t_{1}<t_{2} .
\end{gathered}
$$

Using the identity (see [6])

$$
\Delta_{n h}^{2} f(x)=\sum_{k=1}^{n} k \Delta_{h}^{2} f(x-(n-k) h)+\sum_{k=1}^{n}(n-k) \Delta_{h}^{2} f(x+k h),
$$

$x, h \in \mathbb{R} ; n=2,3, \ldots$, and by (2) and (6) we can prove that

$$
\omega_{2}\left(f, L_{p}^{q} ; \lambda t\right) \leq(1+\lambda)^{2} e^{q(t \lambda)^{2}} \omega_{2}\left(f, L_{p}^{q} ; t\right) \text { for } \lambda, t \geq 0 .
$$

\section{AUXILIARY RESULTS}

In this part, we shall give some fundamental properties of the Gauss-Weierstrass integral operators $W_{n}$ in the spaces $L_{p, 2 q}(\mathbb{R})$.

Lemma 1. The equality

$$
\int_{0}^{\infty} t^{r} e^{-n t^{2}} d t=\frac{1}{2 n^{\frac{r+1}{2}}} \Gamma\left(\frac{r+1}{2}\right)
$$

holds for every $r \in \mathbb{N}_{0}$ and $n>0$. 
Lemma 2. Let $e_{0}(x)=1, e_{1}(x)=x$ and let $\varphi_{x}(t)=t-x$ for $x, t \in \mathbb{R}$ and $k \in \mathbb{N}$. Then,

$$
\begin{gathered}
W_{n}\left(e_{i} ; x\right)=e_{i}(x), \text { for } x \in \mathbb{R}, n \in N, i=0,1 \\
W_{n}\left(\varphi_{x}^{k}(t) ; x\right)=\frac{\left((-1)^{k}+1\right) \Gamma\left(\frac{k+1}{2}\right)}{2 \sqrt{\pi} n^{\frac{k}{2}}} \\
W_{n}\left(\left|\varphi_{x}(t)\right|^{k} \exp \left(q\left|\varphi_{x}(t)\right|^{2} ; x\right)=\sqrt{\frac{n}{\pi}} \frac{\Gamma\left(\frac{k+1}{2}\right)}{(n-q)^{\frac{k+1}{2}}}, n>q+1\right.
\end{gathered}
$$

Lemma 3. Let $f \in L_{p, q}(\mathbb{R})$, with fixed $1 \leq p \leq \infty, q>0$. Then for $n>2 q+1$, we have

$$
\left\|W_{n} f\right\|_{p, 2 q} \leq \sqrt{\frac{n}{n-2 q}}\|f\|_{p, q},
$$

Lemma 3 shows that $W_{n}$ are linear positive operators from $L_{p, q}(\mathbb{R})$ into $L_{p, 2 q}(\mathbb{R})$. Proof. Arguing analogously to the proof of Lemma 2 in [7] we can obtain the above lemma.

Lemma 4. Let $f \in L_{p, q}(\mathbb{R})$ with fixed $1 \leq p \leq \infty$ and $q>0$ and let $n \in N$. Let $f \in L_{\infty, q}^{r}(\mathbb{R})$ with a fixed $r \in \mathbb{N}$. Then $W_{n} f \in L_{\infty, q}^{r}(\mathbb{R})$ and for derivatives of $W_{n} f$ there holds

$$
\left\|\left(W_{n} f\right)^{(k)}\right\|_{\infty, 2 q}=\left\|W_{n} f^{(r)}\right\|_{\infty, 2 q} \leq \sqrt{\frac{n}{n-2 q}}\left\|f^{(k)}\right\|_{\infty, q} .
$$

Proof. For details see [9].

\section{APPROXIMATION RESULTS}

Theorem 5. Let $f \in L_{p, q}(\mathbb{R})$ with fixed $1 \leq p \leq \infty, q>0$ and $n>q+1$. Then we have

$$
\left\|W_{n}(f)-f\right\|_{p, 2 q} \leq \omega_{2}\left(f, L_{p}^{q} ; \frac{1}{\sqrt{n}}\right)\left[\frac{1}{2} \sqrt{\frac{n}{n-q}}+\frac{2 n}{\sqrt{\pi}(n-q)}+\frac{n^{\frac{3}{2}}}{4(n-q)^{\frac{3}{2}}}\right] .
$$

Proof. From (1) and (5) we get

$$
W_{n}(f ; x)-f(x)=\sqrt{\frac{n}{\pi}} \int_{0}^{\infty} \Delta_{t}^{2} f(x) e^{-n t^{2}} d t
$$

for $x \in \mathbb{R}$ and $n>q+1$. By (4) and (8), we get

$$
\left\|W_{n}(f)-f\right\|_{p, 2 q} \leq \sqrt{\frac{n}{\pi}} \int_{0}^{\infty}\left\|\Delta_{t}^{2} f(x)\right\|_{p, q} e^{-n t^{2}} d t
$$




$$
\leq \sqrt{\frac{n}{\pi}} \omega_{2}\left(f, L_{p}^{q} ; \frac{1}{\sqrt{n}}\right) \int_{0}^{\infty}(1+\sqrt{n} t)^{2} e^{-t^{2}(n-q)} d t .
$$

Using Lemma 1, we obtain

$$
\left\|W_{n}(f)-f\right\|_{p, 2 q}=\omega_{2}\left(f, L_{p}^{q} ; \frac{1}{\sqrt{n}}\right)\left[\frac{1}{2} \sqrt{\frac{n}{n-q}}+\frac{2 n}{\sqrt{\pi}(n-q)}+\frac{n^{\frac{3}{2}}}{4(n-q)^{\frac{3}{2}}}\right] .
$$

Thus the theorem is completed.

Corollary 6. Let $f \in L_{p, q}(\mathbb{R})$ with fixed $1 \leq p \leq \infty, q>0$ and $n>q+1$. Then

$$
\lim _{n \rightarrow \infty}\left\|W_{n}(f)-f\right\|_{p, 2 q}=0 \text {. }
$$

Applying Corollary 1, we shall prove the Voronovskaya-type theorem for $W_{n}$.

Theorem 7. Let $f \in L_{q}^{\infty, 2}(\mathbb{R})$ has second derivate at a point $x \in \mathbb{R}$ and with a fixed $q>0$. Then we have

$$
\lim _{n \rightarrow \infty} n\left[W_{n}(f ; x)-f(x)\right]=\frac{f^{\prime \prime}(x)}{4} .
$$

Proof. For $f \in L_{q}^{\infty, 2}$ and $x \in \mathbb{R}$. Then we can use Taylor formula in the form

$$
f(t)=f(x)+f^{\prime}(x)(t-x)+\frac{1}{2} f^{\prime \prime}(x)(t-x)^{2}+\mu(t ; x)(t-x)^{2} \text { for } t \in \mathbb{R},
$$

where $\mu(t)=\mu(t ; x)$ is a function belonging to $L_{q}^{\infty}$ and

$$
\lim _{t \rightarrow x} \mu(t ; x)=\mu(x)=0 .
$$

Using the operator $W_{n},(9)$ and 10$)$, we get

$$
\begin{aligned}
W_{n}(f(t) ; x)= & f(x)+f^{\prime}(x) W_{n}(t-x ; x) \\
& +\frac{1}{2} f^{\prime \prime}(x) W_{n}\left((t-x)^{2} ; x\right)+W_{n}\left(\mu(t) \varphi_{x}^{2}(t) ; x\right) \\
= & f(x)+\frac{1}{4 n} f^{\prime \prime}(x)+W_{n}\left(\mu(t) \varphi_{x}^{2}(t) ; x\right)
\end{aligned}
$$

and by the Hölder inequality and (10), we have

$$
\begin{aligned}
\left|W_{n}\left(\mu(t) \varphi_{x}^{2}(t) ; x\right)\right| & \leq\left(W_{n}\left(\mu^{2}(t) ; x\right)^{\frac{1}{2}} W_{n}\left(\varphi^{4}(t) ; x\right)^{\frac{1}{2}}\right. \\
& =n^{-1}\left(\frac{3}{4} W_{n}\left(\mu^{2}(t) ; x\right)\right)^{\frac{1}{2}} .
\end{aligned}
$$

From properties of $\mu$ and 13 there result that

$$
\lim _{n \rightarrow \infty} W_{n}\left(\mu^{2}(t) ; x\right)=\mu^{2}(x)=0 .
$$


Thus we have

$$
\lim _{n \rightarrow \infty} n W_{n}\left(\mu(t) \varphi_{x}^{2}(t) ; x\right)=0
$$

friom (14) we have desired result.

Theorem 8. Let $f \in L_{q}^{\infty, 2}(\mathbb{R})$ with a fixed $q>0$. Then

$$
\left\|4 n\left[W_{n}(f)-f\right]-f^{\prime \prime}\right\|_{\infty, 2 q} \leq \omega_{1}\left(f^{\prime \prime} ; L_{q}^{\infty} ; \frac{1}{\sqrt{n}}\right)\left[\frac{1}{4}\left(\frac{n}{n-q}\right)^{\frac{3}{2}}+\frac{1}{2 \sqrt{\pi}}\left(\frac{n}{n-q}\right)^{2}\right]
$$

Proof. For $f \in L_{q}^{\infty, 2}$ and $x, t \in \mathbb{R}$ there holds the Taylor-type formula

$$
f(t)=f(x)+f^{\prime}(x)(t-x)+\frac{1}{2} f^{\prime \prime}(x)(t-x)^{2}+(t-x)^{2} I(t, x),
$$

where

$$
I(t, x):=\int_{0}^{1}(1-u)\left[f^{\prime \prime}(x+u(t-x))-f^{\prime \prime}(x)\right] d u
$$

Using operator $W_{n}$, and $(9)-(11)$, we get

$$
W_{n}(f(t) ; x)=f(x)+\frac{1}{4 n} f^{\prime \prime}(x)+W_{n}\left(\varphi_{x}^{2}(t) I(t, x) ; x\right),
$$

which implies that

$$
4 n\left[W_{n}(f ; x)-f(x)\right]-f^{\prime \prime}(x)=n W_{n}\left(\varphi_{x}^{2}(t)|I(t, x)| ; x\right)
$$

for $x \in \mathbb{R}$. Now, applying (4), (7) and (8), we get

$$
\begin{aligned}
|I(t, x)| & \leq \int_{0}^{1}(1-u) \omega_{1}\left(f^{\prime \prime} ; L_{q}^{\infty} ; u|t-x|\right) e^{q x^{2}} d u \\
& \leq \frac{1}{2} \omega_{1}\left(f^{\prime \prime} ; L_{q}^{\infty} ;|t-x|\right) e^{q x^{2}} \\
& \leq \frac{1}{2} \omega_{1}\left(f^{\prime \prime} ; L_{q}^{\infty} ; \frac{1}{\sqrt{n}}\right)(1+\sqrt{n}|t-x|) e^{q x^{2}+q|t-x|^{2}}
\end{aligned}
$$

and next by 2 and 111 , we can write for $x \in \mathbb{R}$ and $n>q+1$,

$$
\begin{aligned}
n \nu_{q}(x) W_{n}\left(\varphi_{x}^{2}(t) I(t, x) ; x\right) \leq & \frac{n}{2} \omega_{1}\left(f^{\prime \prime} ; L_{q}^{\infty} ; \frac{1}{\sqrt{n}}\right) \\
& \times\left\{W_{n}\left((t-x)^{2} e^{q|t-x|^{2}} ; x\right)+\sqrt{n} W_{n}\left(|t-x|^{3} e^{q|t-x|^{2}} ; x\right\}\right. \\
= & \omega_{1}\left(f^{\prime \prime} ; L_{q}^{\infty} ; \frac{1}{\sqrt{n}}\right)\left[\frac{1}{4}\left(\frac{n}{n-q}\right)^{\frac{3}{2}}+\frac{1}{2 \sqrt{\pi}}\left(\frac{n}{n-q}\right)^{2}\right] .
\end{aligned}
$$

Now the estimate 15 is obtained by $(16)$, the last inequality and $(3)$. 
Theorem 9. Let $f \in L_{q}^{\infty, r}$, with fixed $q>0$ and $r \in \mathbb{N}$. Then

$$
\begin{aligned}
\left\|W_{n}^{(r)}(f)-f^{(r)}\right\|_{\infty, 2 q} \leq & \omega_{2}\left(f^{(k)} ; L_{q}^{\infty} ; \frac{1}{\sqrt{n}}\right) \\
& \times\left(\sqrt{\frac{n}{n-2 q}}+\frac{n}{(n-2 q) \sqrt{\pi}}+\frac{1}{4}\left(\frac{n}{n-2 q}\right)^{\frac{3}{2}}\right)
\end{aligned}
$$

for $n>2 q+1$.

Proof. If $f \in L_{q}^{\infty, r}$, then for r-th derivative of $W_{n}(f)$ we have by Lemma $4,(9)$ and 10):

$$
\begin{aligned}
W_{n}^{(r)}(f ; x)-f^{(r)}(x) & =\sqrt{\frac{n}{\pi}} \int_{-\infty}^{\infty}\left[f^{(r)}(x+t)-f^{(r)}(x)\right] e^{-n t^{2}} d t \\
& =\sqrt{\frac{n}{\pi}} \int_{0}^{\infty}\left[\Delta_{t}^{2} f^{(r)}(x-t)\right] e^{-n t^{2}} d t .
\end{aligned}
$$

from this and by (4), (8) and Lemma 1 we deduce that

$$
\begin{aligned}
& \left\|W^{(r)} r(f ; x)-f^{(r)}(x)\right\|_{\infty, 2 q} \leq \sqrt{\frac{n}{\pi}} \int_{0}^{\infty} \omega_{2}\left(f^{(r)} ; L_{q}^{\infty} ; t\right) e^{-(n-q) t^{2}} d t \\
& \leq \omega_{2}\left(f^{(r)} ; L_{q}^{\infty} ; \frac{1}{\sqrt{n}}\right) \sqrt{\frac{n}{\pi}} \int_{0}^{\infty}(1+\sqrt{n} t)^{2} e^{-t^{2}(n-2 q)} d t \\
& =\omega_{2}\left(f^{(r)} ; L_{q}^{\infty} ; \frac{1}{\sqrt{n}}\right) \\
& \times \sqrt{\frac{n}{\pi}}\left[\int_{0}^{\infty} e^{-t^{2}(n-2 q)} d t+2 \sqrt{n} \int_{0}^{\infty} t e^{-t^{2}(n-2 q)} d t+n \int_{0}^{\infty} t^{2} e^{-t^{2}(n-2 q)} d t\right] \\
& =\omega_{2}\left(f^{(r)} ; L_{q}^{\infty} ; \frac{1}{\sqrt{n}}\right) \\
& \times\left(\sqrt{\frac{n}{n-2 q}}+\frac{n}{(n-2 q) \sqrt{\pi}}+\frac{1}{4}\left(\frac{n}{n-2 q}\right)^{\frac{3}{2}}\right)
\end{aligned}
$$

for $n>2 q+1$, which yields the estimate 17 .

\section{REFERENCES}

[1] Anastassiou, G. A.,Aral, A., On Gauss-Weierstrass type integral oprators. Demonstratio Mathematica 43(2010), no.4, 841-849. 
[2] Becker , M., Kucharski, D., Nessel, R. J., Global approximation theorems for the SzaszMirakyan operators in exponential weight spaces, in Linear Spaces and Approximation, Proc. Conf. Oberwolfach, 1977), Birkhauser Verlag, Basel (1978), pp. 319-333.

[3] Butzer, P.L., Nessel, R.J. Fourier Analysis and Approximation, I,II; Birkhauser, Basel and Academic Press, New York, 1971

[4] Deeba, E., Mohapatra, R.N., Rodriguez, R. S., On the degree of approximation of some singular integrals, Rend. Mat. Appl. 7(1988), 345-355.

[5] Ditzian, Z., Totik, V., Moduli of Smoothness, Springer-Verlag, New-York, 1987.

[6] Ibragimov, I. I, Theory of Approximation by Entire Functions, in Russian, Baku, 1979.

[7] Leśniewicz, A., Rempulska, L., Wasiak, J., Approximation properties of the Picard singular integral in exponential weight spaces, Publ. Mat. 40(1996), no.2, 233-242.

[8] Mohapatra, R.N., Rodriguez, R.S., On the rate of convergence of singular integrals for Hölders continuous functions.Math. Nachr. 149(1990), 117-124.

[9] Rempulska, L. and Tomczak, K., On some properties of the Picard operators, Arch. Math. (Brno), 45(2009), 125-135.

Current address: Department of Mathematics, Faculty of Science and Arts, Kirikkale University, Yahsihan, 71450 Kiriklale, Turkey

E-mail address: basaryilmaz77@yahoo.com

ORCID Address: http://orcid.org/0000-0003-3937-992X 